\title{
2-Arylthiazolidine-4-carboxylic acid amides (ATCAA) target dual pathways in cancer cells: 5'-AMP-activated protein kinase (AMPK)/mTOR and PI3K/Akt/mTOR pathways
}

\author{
CHIEN-MING LI ${ }^{1,2^{*}}$, RAMESH NARAYANAN ${ }^{1 *}$, YAN LU $^{3}$, EUNJU HURH $^{2}$, CHRISTOPHER C. COSS ${ }^{1}$, \\ CHRISTINA M. BARRETT ${ }^{1}$, DUANE D. MILLER ${ }^{1,3}$ and JAMES T. DALTON ${ }^{1,2}$ \\ ${ }^{1}$ GTx Inc., Memphis, TN 38163; ${ }^{2}$ Division of Pharmaceutics, College of Pharmacy, The Ohio State University, \\ Columbus, OH 43210; ${ }^{3}$ Department of Pharmaceutical Sciences, University of Tennessee \\ Health Science Center, Memphis, TN 38163, USA
}

Received May 25, 2010; Accepted June 25, 2010

DOI: 10.3892/ijo_00000754

\begin{abstract}
Phosphatidylinositol-3-kinase (PI3K)/Akt and 5 '-AMP-activated protein kinase (AMPK) are attractive targets for anti-cancer drug development. Inhibition of Akt or activation of AMPK is cytotoxic to human cancer cells in vitro and in vivo. We previously demonstrated that 2arylthiazolidine-4-carboxylic acid amides (ATCAA) are effective cytotoxic agents in prostate and melanoma cancer cell lines, with $\mathrm{IC}_{50}$ values in the low/sub micromolar range. Using in vitro and in vivo studies, we further characterized the anti-cancer efficacy and mechanism of action of ATCAA-10, a potent lead. ATCAA-10 exhibited equal potency on both MES/SA and P-glycoprotein over-expressing multidrug resistant MES/SA/Dx5 cells, suggesting that ATCAA-10 may overcome multiple drug resistance. Cell-free kinase binding assays excluded the direct binding of ATCAA-10 to several kinases, including IGF-1R, EGFR, FGFR and PDGFR. However, in A549 and HeLa cells, ATCAA-10 effectively dephosphorylated Akt, with concomitant phosphorylation of AMPK. Determination of intracellular ATP and AMP concentrations revealed that ATCAA-10 activated AMPK by altering the intracellular AMP/ATP ratio. ATCAA-10 exhibited favorable pharmacokinetic properties in both mice and rats, including low clearance, low hepatic extraction rate, moderate volume of distribution and long half-life. In addition, ATCAA-10 inhibited A549 tumor xenograft growth with $46 \%$ tumor growth inhibition (TGI) at $20 \mathrm{mg} / \mathrm{kg}$ dose. Taken together; these results suggest that ATCAA-10
\end{abstract}

Correspondence to: Dr James T. Dalton, GTx Inc., 3 Dunlap Street, Memphis, TN 38163, USA

E-mail: jdalton@gtxinc.com

*Contributed equally

Key words: 2-arylthiazolidine-4-carboxylic acid amides, PI3K/ Akt/mTOR, AMP-activated protein kinase, pharmacokinetics, xenograft modulates the activity of two signaling pathways, PI3K/ AKT/mTOR and AMPK/mTOR, resulting in the inhibition of cancer cell growth.

\section{Introduction}

The phosphatidylinositol 3-kinase/Akt (PI3K/Akt) pathway regulates many cellular functions and is aberrantly activated in many human malignancies. Eight isoforms of PI3K exist, which are divided into classes I, II, and III PI3Ks. Class I PI3Ks have drawn the most attention due to their roles in cell survival (1). Class I PI3K is further divided into classes $\mathrm{Ia}$ and $\mathrm{Ib}$; with class Ia activated by receptor tyrosine kinases and class Ib activated by G-protein-couple receptors (GPCRs). Once activated, class I PI3Ks rapidly phosphorylate phosphatidylinositol $(4,5)$-bisphosphate (PIP2) to phosphatidylinositol-3, 4, 5-trisphosphate (PIP3). Signaling proteins, such as PDK1 and Akt, bind to PIP3 via their pleckstrin homology domain (PH domain) with subsequent phosphorylation of Akt by PDK1 or other kinases $(2,3)$. PI3K can be inactivated by the tumor suppressor protein, phosphatase and tensin homolog (PTEN), which converts PIP3 back to PIP2, hampering the lipid signaling. Loss or mutation of PTEN constitutively activates Akt leading to increased cellular proliferation and cancer (4). Thus, inhibition of PI3K/Akt signaling provides a promising strategy to prevent and treat cancers, such as prostate cancer (5). There are many substrates of Akt involved in various physiological roles. While glycogen synthase kinase 3 (GSK3) regulates glucose metabolism and cell cycle-regulatory proteins $(6,7)$, mTOR, another substrate, inhibits tuberous sclerosis complex 2 (TSC2), resulting in increased mTORC1 activity (8). Another proliferative protein, S6 ribosomal protein functions downstream of mTOR. Inhibition of the PI3K/Akt/mTOR signaling cascade is therefore viewed as a logical means to inhibit cell growth and treat cancer $(9,10)$.

AMP-activated protein kinase (AMPK), a serine/threonine kinase, consists of a heterotrimeric complex containing a catalytic $\alpha$ subunit and regulatory $\beta$ and $\gamma$ subunits (11). AMP binds to AMPK allosterically to facilitate phosphorylation on Thr-172, an effect that is mediated by the tumor suppressor 
protein, LKB1 (12). Previous studies have shown that the allosteric activation of AMPK is antagonized by ATP, suggesting that both AMP and ATP bind to the same site (13) and that AMPK can be activated by ATP depletion. Overall, activation of AMPK is associated with an increased intracellular AMP/ATP ratio (14). AMPK activation, by synthetic molecules, such as 2-deoxyglucose (2DG) or 5-aminoimidazole-4-carboxyamide ribonucleotide (AICAR), has been shown to activate TSC2, which in turn inhibits the downstream target of mTOR, S6K $(15,16)$. Activated AMPK also positively regulates p53 and p21, which are important proteins that control growth arrest and apoptosis. In addition, AMPK regulates acetyl-CoA carboxylase (ACC) and glycerol phosphate acyltransferase for fatty acid and triglyceride synthesis, respectively (17). mTOR is the downstream target of the PI3K/Akt and AMPK pathways, suggesting that both $\mathrm{PI} 3 \mathrm{~K} / \mathrm{Akt} / \mathrm{mTOR}$ and AMPK/mTOR pathways are potential therapeutic targets for cancer $(18,19)$.

We previously studied the structure-activity relationship (SAR) of a series of 2-arylthiazolidine-4-carboxylic acid amides (ATCAA) in prostate and melanoma cancer cells (20). ATCAA-10, our most potent compound, exhibited submicromolar $\mathrm{IC}_{50}$ values in cytotoxicity assays and induced severe sub-G1 phase arrest. In this study, we demonstrate that ATCAA-10 exhibited dual effects on PI3K/Akt/mTOR and AMPK/mTOR pathways. ATCAA-10 inactivated PI3K/Akt and activated AMPK, resulting in synergistic inactivation of mTOR/S6K. We also studied the effects of ATCAA-10 on the upstream and downstream pathways of AMPK. Pharmacokinetics, toxicity and in vivo efficacy (human lung cancer A549 cells) were also examined in this report.

\section{Materials and methods}

Cell culture and cytotoxicity assay. Cell lines were obtained from ATCC (American Type Culture Collection, Manassas, VA, USA), while cell culture supplies were purchased from Cellgro Mediatech (Herndon, VA, USA). We examined the antiproliferative activity of ATCAA-10 in four human prostate cancer cell lines (LNCaP, DU 145, PC-3, and PPC-1), two human lung cell lines (A549 and H1299) and one cervical cancer cell line (HeLa). Human sarcoma cell line MES-SA and its doxorubicin-resistant cell line that over-expresses P-glycoprotein (P-gp), MES-SA/Dx5, were used as MDR models. All prostate cancer cell lines were cultured in RPMI1640 medium. Lung cancer cells and sarcoma cells were cultured in Dulbecco's modified Eagle's medium (DMEM). HeLa cells were grown in Eagle's MEM medium. MES-SA and MES-SA/Dx5 cells were grown in McCoy's 5A medium. All cell media were supplemented with $10 \%$ fetal bovine serum (FBS). The cytotoxic potential of ATCAA-10 was evaluated using sulforhodamine B (SRB) assay after $96 \mathrm{~h}$ after treatment. Percentage of cell survival was plotted against drug concentrations and the $\mathrm{IC}_{50}$ values (concentration that inhibited cell growth by $50 \%$ of untreated control) were obtained by nonlinear regression analysis using WinNonlin (Pharsight Corp., Mountain View, CA).

Cell treatment and immunoblotting. A549 and HeLa cells were grown in DMEM and Eagle's MEM medium containing
$10 \%$ FBS, respectively, in humidified, $37^{\circ} \mathrm{C}$ chambers with $5 \% \mathrm{CO}_{2}$. A549 cells were treated with $10 \mu \mathrm{M}$ ATCAA-10 for 2, 4 and $6 \mathrm{~h}$. Varying concentrations of ATCAA-10 were used to treat A549 and HeLa cells for 6 and $4 \mathrm{~h}$, respectively. Cells were washed twice by cold phosphate-buffered saline (PBS) and lysed in lysis buffer [20 mM Tris-HCl (pH 7.5), $150 \mathrm{mM} \mathrm{NaCl}, 1 \mathrm{mM} \mathrm{Na} 2$ EDTA, $1 \mathrm{mM}$ EGTA, $1 \%$ Triton, $2.5 \mathrm{mM}$ sodium pyrophosphate, $1 \mathrm{mM}$ B-glycerophosphate, $1 \mathrm{mM} \mathrm{Na} \mathrm{VO}_{4}, 1 \mu \mathrm{g} / \mathrm{ml}$ leupeptin] incubated on ice for $30 \mathrm{~min}$. The lysates were centrifuged at $14,000 \mathrm{x}$ g for $20 \mathrm{~min}$ and supernatants were collected. Proteins $(50 \mu \mathrm{g})$ were resolved in a 4-20\% gradient Tris-glycine gel, and transferred to nitrocellulose. Blots were probed with antibodies to phosphoThr172-AMPK, total-AMPK, phospho-Ser473-AKT, totalAKT, phospho-Ser9-GSK3ß, total-GSK3ß, phospho-Ser235/ 236-S6 ribosomal protein, or total-S6 ribosomal protein. All antibodies were purchased from Cell Signaling (Beverly, MA). Immunoblots were developed using ECL Plex CyDye conjugated with goat anti-rabbit IgG-Cy5 (GE Healthcare, Waukesha, WI), followed by detection with fluorescence imaging system (Typhoon 8600, GE Healthcare).

Nucleotide extraction and FPLC analysis. HeLa cells were treated with vehicle, ATCAA-10 $(10 \mu \mathrm{M})$ and PD98059 $(100 \mu \mathrm{M})$ for $4 \mathrm{~h}$. Following treatments, cells were washed quickly in PBS and the cell pellets were obtained by centrifugation at 14,000 x g for $20 \mathrm{~min}$. Perchloric acid $\left(\mathrm{HClO}_{4}\right.$, $400 \mu 1$ of $0.2 \mathrm{M}$ ) was added and incubated on ice for $10 \mathrm{~min}$. $\mathrm{KOH}(100 \mu 1$ of $1 \mathrm{~N})$ was added to neutralize the $\mathrm{pH}$ to $\sim 7.0$. Macromolecules were removed using an ultrafiltration method (microconcentrator) (Microcon, Bedford, MA) with a molecular cutoff size of $30 \mathrm{kDa}$. Nucleotides were separated by ion-exchange chromatography on a Mono Q 5/50 GL column run on Monitor UPC-900 system (GE Healthcare). The column was equilibrated with $10 \mathrm{mM}$ Tris- $\mathrm{HCl}, \mathrm{pH} 8.0$, and developed with a linear gradient to $10 \mathrm{mM}$ Tris- $\mathrm{HCl}$ with $1.5 \mathrm{M} \mathrm{NaCl}$ at a flow rate $1 \mathrm{ml} / \mathrm{min}$. Nucleotides were detected by their absorance at $254 \mathrm{~nm}$. Peak heights were used to quantify and calculate the AMP:ATP ratios.

AMPK activity. Active AMPK $(\alpha 1 / \beta 1 / \gamma 1)$ enzyme and commercial AMPK kinase assay kit were purchased from CycLex Co., Ltd. (Nagano, Japan) for measuring AMPK activity. Pure AMPK enzyme (40 ng) was incubated with ATCAA-10 $(10 \mu \mathrm{M})$ in kinase buffer at $30^{\circ} \mathrm{C}$ for $40 \mathrm{~min}$. AMP $(10 \mu \mathrm{M})$ was used as the positive control. The absorbance at wavelength $450 \mathrm{~nm}$ was used to represent the AMPK activity compared to the vehicle control.

Cell-free purified enzyme activity assay. ATCAA-10 $(20 \mu \mathrm{M})$ was used to examine the inhibition of enzyme activity. Potency $\left(\mathrm{IC}_{50}\right)$ was determined using full concentration range of ATCAA-10. Human receptor tyrosines kinases (RTKs), including IGF-1R, EGFR, FGFR, and PDGFR, were examined. DMSO $(0.5 \%)$ was used as negative control, and staurosporine was used as positive control for all RTKs. Each RTK (h) (10 mU) was incubated with $8 \mathrm{mM} \mathrm{3-(N-morpholino)}$ propanesulfonic acid (MOPS), $0.2 \mathrm{mM}$ EDTA, $10 \mathrm{mM}$ $\mathrm{MnCl}_{2}, 0.1 \mathrm{mg} / \mathrm{ml}$ poly(Glu:Tyr, 4:1), $10 \mathrm{mM} \mathrm{Mg}$ acetate, $\left[\gamma^{-33} \mathrm{P}\right]$-ATP (activity at $500 \mathrm{cpm} / \mathrm{pmol}$, concentration as 
required), and ATCAA-10. The reaction was initiated by adding Mg/ATP mix in a final reaction volume of $25 \mu 1$. After 40-min incubation at room temperature, the reaction was stopped by adding $5 \mu \mathrm{l}$ of $3 \%$ phosphoric acid solution. Ten microliters of the reaction was then spotted onto a Filtermat $\mathrm{A}$ and washed three times for $5 \mathrm{~min}$ in $75 \mathrm{mM}$ phosphoric acid and once in methanol prior to drying and scintillation counting. Percentage of kinase activities were compared with vehicle.

Pharmacokinetic study. All animal studies were conducted under the auspices of a protocol reviewed and approved by the Institutional Laboratory Animal Care and Use Committee of either The University of Tennessee or The Ohio State University. Male ICR mice ( $\mathrm{n}=3$, each time-point) 6-8 weeks of age were purchased from Harlan Inc. (Indianapolis, IN). ATCAA-10 (10 mg/kg) was dissolved in PEG300/DMSO (1/4) and administered by a single i.v. injection into their tail vein. Blood samples were collected in heparinized tubes via cardiac puncture under isoflurane anesthesia at 2,5,15, and 30 min, 1, 2, 4, 8, 16, and $24 \mathrm{~h}$ after administration. Plasma samples were collected by centrifuge at $8,000 \mathrm{~g}$ for $5 \mathrm{~min}$. All plasma samples were stored immediately at $-80^{\circ} \mathrm{C}$ until analyzed.

Male Sprague-Dawley rats $(n=4 ; 254 \pm 4 \mathrm{~g})$ were purchased from Harlan Inc. (Indianapolis, IN). Rat thoracic jugular vein catheters (JVC) were purchased from Braintree Scientific Inc. (Braintree, MA). On arrival to the animal facility, the animals were acclimated for 3 days in a temperature-controlled room $\left(20-22^{\circ} \mathrm{C}\right)$ with a 12 -h light/dark cycle before any treatment. ATCAA-10 was administered intravenously into the JVC at $2.5 \mathrm{mg} / \mathrm{kg}$ (in DMSO/PFG300, 1/4). Equal volume of heparinized saline was injected to replace the removed blood and rinse the catheter. Blood samples $(250 \mu \mathrm{l})$ were collected via the JVC at 10, 20, 30 min, and 1, 2, 4, 8, 12, $24 \mathrm{~h}$. Plasma samples were obtained as previously described. An aliquot of plasma was used to measure plasma insulin levels using the rat insulin ELISA kit (Crystal Chem Inc., Downers Grove, IL) following the manufacturer's instructions. Whole blood samples $(5 \mu 1)$ were also collected and used to measure blood glucose levels using a commercial glucose meter (ACCU-CHEK, Hamilton, New Zealand).

ATCAA-10 was extracted from $100 \mu 1$ of plasma with $200 \mu \mathrm{l}$ of acetonitrile (ACN) containing $100 \mathrm{nM}$ internal standard (ATCAA analog). The samples were thoroughly mixed, centrifuged, and the organic extract was transferred to autosampler for LC-MS/MS analysis. The PK parameters were determined using non-compartment analysis (WinNonlin, Pharsight Corp., Mountain View, CA).

LC-MS/MS analytical method. Aliquots $(10 \mu 1)$ of the supernatant were injected into the HPLC system (Model 1100 Series Chemstation, Agilent Technology Co., Santa Clara, CA). A narrow-bore C4 column (2.1x150 mm, $5 \mu \mathrm{m}$, Varian Inc, Palo Alto, CA) was used to separate ATCAA-10 from plasma matrix. Gradient mode was used to achieve separation of the analyte using mixtures of mobile phase A $5 \% / 95 \%$ acetonitrile $/ \mathrm{H}_{2} \mathrm{O}$ containing $0.1 \%$ formic acid) and mobile phase $\mathrm{B}\left(95 \% / 5 \%\right.$ acetonitrile $/ \mathrm{H}_{2} \mathrm{O}$ containing $0.1 \%$ formic acid) at a flow rate of $300 \mu \mathrm{l} / \mathrm{min}$. Mobile phase A was used at $80 \%$ from 0 to $1 \mathrm{~min}$ followed by a linearly programmed gradient to $100 \%$ of mobile phase B within $3 \mathrm{~min}, 100 \%$ of mobile phase B was maintained for 1 min before a quick ramp to $80 \%$ mobile phase A. Mobile phase A was continued for another $8 \mathrm{~min}$ towards the end of analysis.

A triple-quadruple mass spectrometer (API Qtrap ${ }^{\mathrm{TM}}$ Applied Biosystems/MDS SCIEX, Concord, Ontario, Canada) operating with a TurboIonSpray source was used. Multiple reaction monitoring $(\mathrm{MRM})$ mode, scanning $\mathrm{m} / \mathrm{z}$ 523.0 $\rightarrow 196.3$ (ATCAA-10) and m/z 434.0 $\rightarrow 266.0$ (IS) was used for quantitation.

A549 tumor xenograft studies. A549 cells $\left(8 \times 10^{7}\right.$ per ml) were prepared in phenol-red-free growth media containing $10 \%$ FBS, and mixed with Matrigel (BD Biosciences, San Jose, CA) at 1:1 ratio. Tumors were established by injecting $100 \mu 1$ of the mixture $\left(4 \times 10^{6}\right.$ cells per animal) subcutaneously (s.c.) into the flank of 6-8-week-old male athymic nude mice. Length and width of tumors were measured and the tumor volume $\left(\mathrm{mm}^{3}\right)$ was calculated by the formula: $\pi / 6 \times \mathrm{L} \mathrm{x} \mathrm{W}^{2}$, where length (L) and width (W) were determined in $\mathrm{mm}$. When the tumor volumes reached $200 \mathrm{~mm}^{3}$, the animals bearing A549 tumors were treated with vehicle [Captex200/Tween-80 (1/4)], ATCAA-10 (5, 10, 15 and $20 \mathrm{mg} / \mathrm{kg})$ intraperitorally twice a week for 31 days.

\section{Results}

ATCAA-10 inhibits the growth of human cancer cell lines and multidrug-resistant cancer cell lines. The ability of ATCAA-10 (Fig. 1A) to inhibit the growth of cancer cell lines was evaluated using the SRB assay. As shown in Table I, ATCAA-10 inhibited the growth of several human cancer cell lines, including four prostate cancer cell lines and two lung cancer cell lines, with $\mathrm{IC}_{50}$ values in the low micro-molar range. In addition, the effect of ATCAA-10 in the doxorubicin resistant cell line MES-SA/Dx5 was also evaluated (Table I). ATCAA-10 exhibited a smaller resistance factor (1.7-fold) compared to the positive control, vinblastine, a P-gp substrate (9.3-fold). In addition, the slope of doseresponse curve varied for vinblastine, but not for ATCAA-10 (data not shown). These data indicate that ATCAA-10 may overcome P-gp mediated drug resistance.

ATCAA-10 inhibits Akt phosphorylation and activates AMPactivated protein kinase (AMPK). We then examined the effect of ATCAA-10 on the PI3K/Akt pathway. Fig. 1B shows that ATCAA-10 $(10 \mu \mathrm{M})$ decreased phospho-Akt levels without changing total-Akt levels in A549 cells in a time-dependent manner. At $6 \mathrm{~h}$, the ratio of phospho-Akt to total-Akt was lower compared to the control. Simultaneously phosphoAMPK was induced by ATCAA-10 at $6 \mathrm{~h}$ (Fig. 1B), suggesting that ATCAA-10 impinges on both PI3K/Akt and AMPK pathways. The treatment time $(6 \mathrm{~h})$ was fixed for future dose-response studies and the dose-dependent effects of ATCAA-10 were examined (Fig. 1C) in A549 cells. Phosphorylation of a downstream target of Akt, phospho-Ser235/ 236-S6 ribosomal protein, was also greatly reduced following ATCAA-10 treatment (Fig. 1C). The total protein level of S6 ribosomal protein remained unchanged. Modulation of 
A<smiles>COc1cc(C2NC(C(N)=O)CS2)cc(OC)c1OC(N)=O</smiles>

C $\begin{array}{lllllllll}\text { Conc. }(\mu \mathrm{M}) & 0 & 0.03 & 0.1 & 0.3 & 1 & 2 & 5 & 10\end{array}$

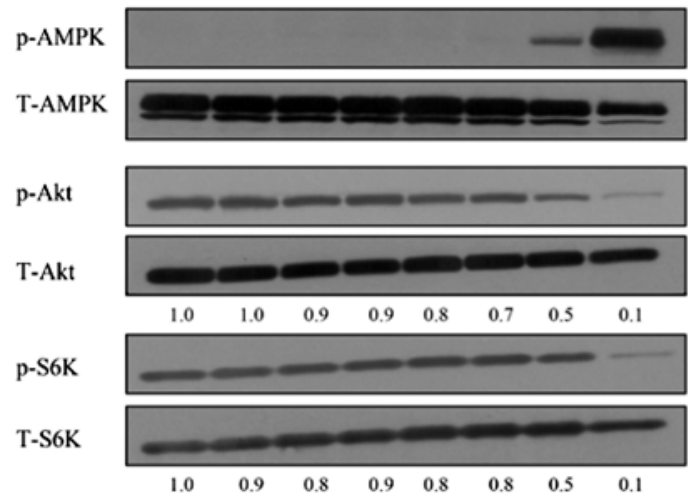

B

D
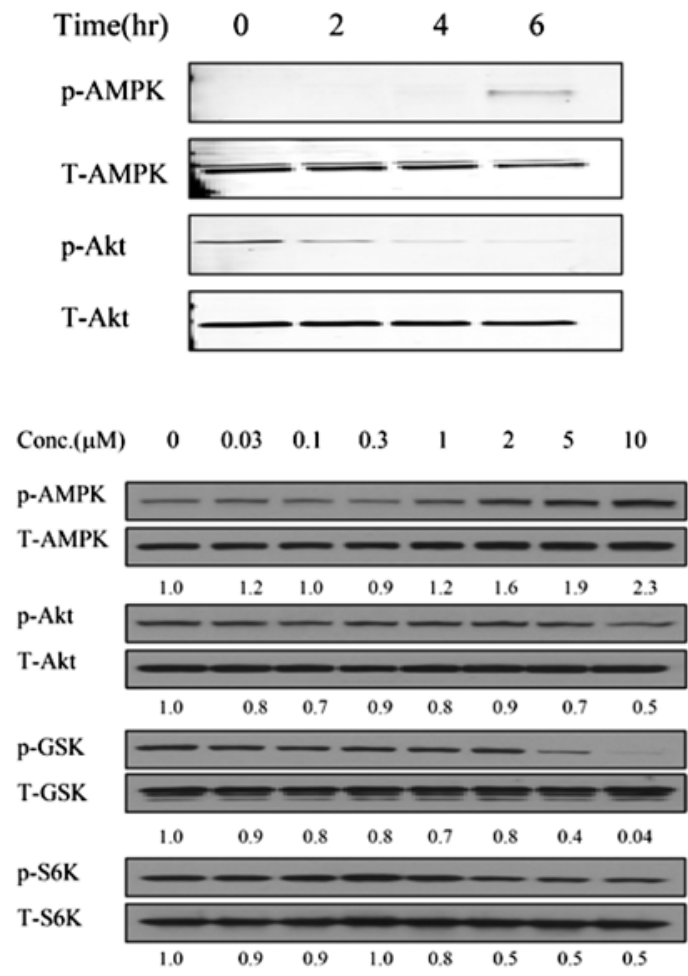

Figure 1. ATCAA-10 activated AMPK and deactivated PI3K/Akt pathways. (A) The structure of ATCAA-10. (B) Effects of ATCAA-10 (10 $\mu$ M) in A549 cells at indicated time-points. (C) Dose-response of ATCAA-10 in A549 cells after 6-h treatment. (D) Dose-response of ATCAA-10 in HeLa cells after 4-h treatment. The number under blots indicate the ratio of phosphor-protein and total-protein related to the control.

Table I. Potency of ATCAA in inhibiting cell growth in vitro. ${ }^{\mathrm{a}}$

\begin{tabular}{ll}
\hline Tumor cells & $\mathrm{IC}_{50}, \mu \mathrm{M}$ \\
\hline Prostate cancer & \\
LNCaP & $0.8 \pm 0.1^{\mathrm{b}}$ \\
PC-3 & $1.8 \pm 0.1^{\mathrm{b}}$ \\
Du-145 & $1.7 \pm 0.1^{\mathrm{b}}$ \\
PPC-1 & $0.4 \pm 0.1^{\mathrm{b}}$
\end{tabular}

Lung cancer

A549

$1.1 \pm 0.2$

H1299

$0.4 \pm 0.1$

Cervical cancer

HeLa

$1.4 \pm 0.1$

Human uterine sarcoma

MES-SA

$1.0 \pm 0.1$

MES-SA/Dx5

$1.7 \pm 0.1(1.7 \mathrm{X})$

MES-SA (vinblastine)

$0.0014 \pm 0.0001$

MES-SA/Dx5 (vinblastine)

$0.013(9.3 \mathrm{X})$

${ }^{a}$ Cell growth inhibition was measured by SRB assay after 96-h treatment. The numbers in parenthesis are folds of resistance factor based on the $\mathrm{IC}_{50}$ ratio when compared with the parent cell line (MES-SA). ${ }^{b}$ Values represent the mean \pm SD of triplicates. Previously reported in ref. 20 .
phospho-Ser9-GSK3ß, another downstream target of Akt, was also examined in A549 cells; however, the signal was under the detection limit (data not shown).

HeLa cells, which are known to be LKB1-null, were then used to confirm the mechanism of action of ATCAA-10 via PI3K/Akt and AMPK pathways. As shown in Fig. 1D, ATCAA-10 exhibited the same effect on Akt and AMPK in HeLa cells as observed in A549 cells, with phosphor-AMPK levels induced more than 2-fold and a concomitant decrease in phosphor-Akt levels. Phospho-Ser9-GSK3ß and phosphoSer235/236-S6 ribosomal protein levels were also decreased in a dose-dependent manner, indicating that the effects of ATCAA-10 on Akt and AMPK were not cell type-dependent.

In previous studies, we demonstrated that ATCAA-10 failed to target LPA receptors (20). In an attempt to identify the molecular target, we examined the interaction of ATCAA10 with several receptor tyrosine kinases (RTKs), including IGF-1R, EGFR, FGFR and PDGFR in this study. ATCAA-10 exhibited $\mathrm{IC}_{50}$ values $>20 \mu \mathrm{M}$ for all these kinases (Table II), suggesting that ATCAA-10 did not appreciably interact with these potential targets. ATCAA-10 did not target PDK1, which is the direct upstream of Akt. In addition, ATCAA-10 did not reduce activities on PI3K isoforms $(\beta, \gamma, \delta)$ and Akt isoforms $(\alpha, \beta, \gamma)$ (Table II). The major target causing deactivation of Akt by ATCAA-10 remains unclear.

ATCAA-10 activates AMPK by decreasing intracellular $A M P / A T P$ ratio. An FPLC method was developed to measure intracellular AMP, ADP and ATP levels (Fig. 2A). ATCAA-10 $(10 \mu \mathrm{M})$ treatment reduced AMP and ATP levels, while ADP 
Table II. Inhibition of pure enzyme by ATCAA-10. ${ }^{\mathrm{a}}$

Enzyme

Activity, $\% \pm \mathrm{SD}$

\begin{tabular}{lc}
\hline Receptor tyrosine kinase & \\
IGF 1R & 100 \\
EGFR (L858R) & 46 \\
EGFR (L861Q) & 65 \\
EGFR (T790M) & 66 \\
FGFR 1 & 82 \\
FGFR (V561M) & 77 \\
PDGFR $\alpha$ & 117 \\
PDGFRß & 94 \\
PDK1 & $108 \pm 4$ \\
PI3-K p110 & $105 \pm 2$ \\
$(ß)$ & $94 \pm 4$ \\
$(\gamma)$ & $85 \pm 3$ \\
$(\delta)$ & \\
Akt & \\
$(\alpha)$ & $92 \pm 6$ \\
$(ß)$ & $72 \pm 5$ \\
$(\gamma)$ & $90 \pm 3$ \\
\hline aACAA-10 (20 $\mu$ M) was used to test inhibition of enzyme activity \\
in cell-free system. Receptor tyrosine kinases were tested in duplicate, \\
and mean values are presented. PDK1, PI3K and Akt were tested in \\
triplicate (mean \pm SD). \\
\hline
\end{tabular}

levels remained the same compared to the control (Fig. 2B). ATCAA-10 reduced ATP levels more than AMP levels, resulting in an increase in the ratio of intracellular AMP/ATP (Fig. 2C). PD98059 was used as a positive control; however, PD98059 increased the ratio of AMP/ATP by reducing ATP levels without altering the AMP levels. Pure and active AMPK enzyme was used to understand the direct effect of ATCAA-10 on AMPK, if any. Interestingly, ATCAA-10 failed to stimulate AMPK (Fig. 2D), suggesting that ATCAA-10 induces phosphorylation of AMPK indirectly by increasing the ratio of intracellular AMP/ATP, but not by directly stimulating AMPK.

Pharmacokinetic study (PK) of ATCAA-10 in mice and rats. Since ATCAA-10 had an interesting in vitro pharmacologic profile, we determined its PK properties before proceeding to tumor xenograft studies. The plasma concentration vs. time profiles in mice and rats given a single i.v. bolus dose of $10 \mathrm{mg} / \mathrm{kg}$ ATCAA-10 are shown in Fig. 3. The PK parameters of ATCAA-10 in mice and rats are summarized in Table III, indicating that ATCAA-10 exhibited favorable PK properties in both mice and rats. In mice, ATCAA-10 had a long elimination half-life (311 min), moderate volume of distribution at steady state $(2.9 \mathrm{l} / \mathrm{kg})$, but high total plasma clearance $(34 \mathrm{ml} / \mathrm{min} / \mathrm{kg}$, hepatic extraction rate is 0.68 , if the hepatic plasma flow rate is $50 \mathrm{ml} / \mathrm{min} / \mathrm{kg}$ in mice). The plasma concentration vs. time profile was also examined in mice given single dose by intraperitoneal (i.p.) route (Fig. 3A). Compared to the area under the curve (AUC) by i.v. administration, the bioavailability of i.p. administration was $93 \%$, indicating that the i.p. route provided an efficient and easy method of
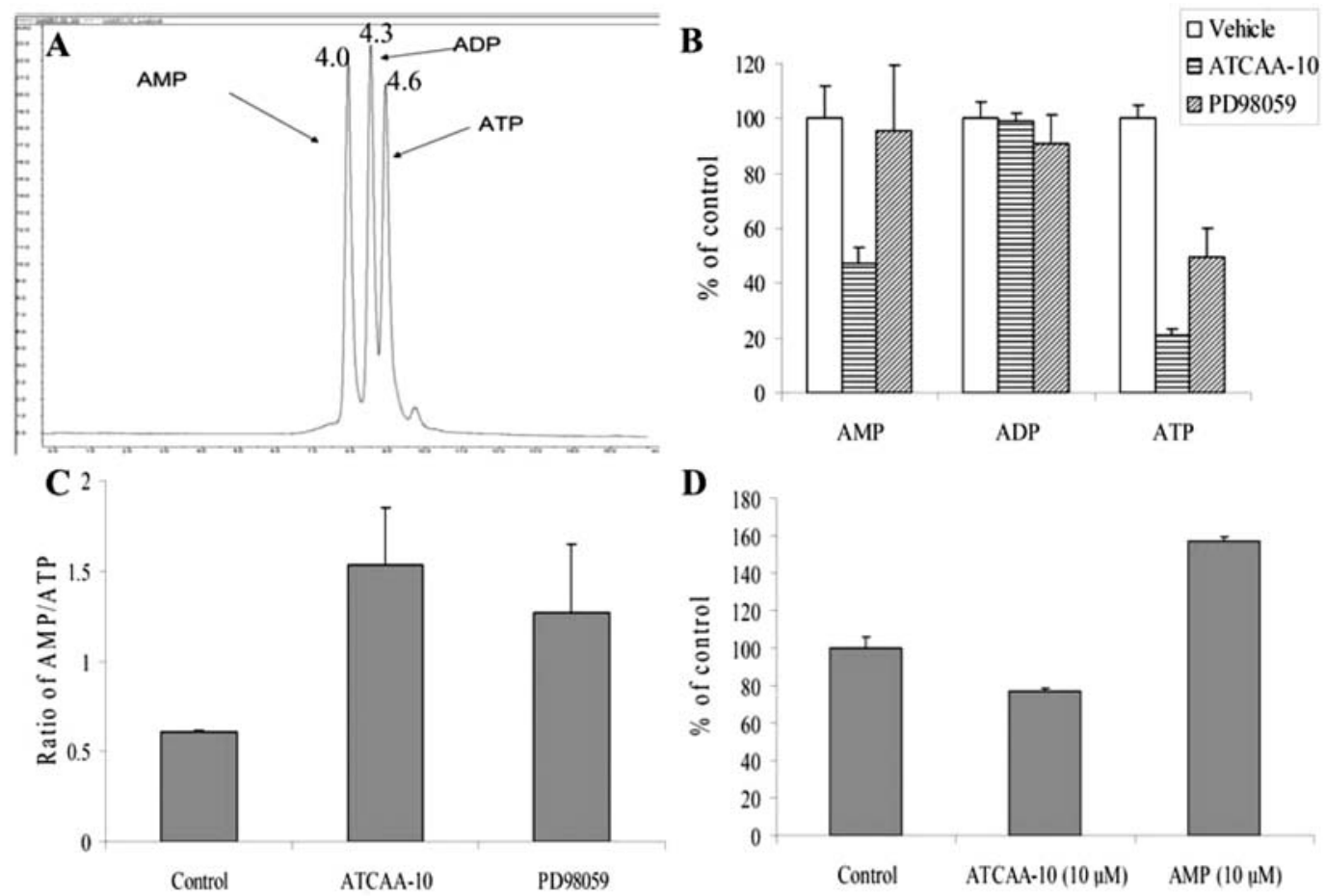

Figure 2. ATCAA-10 stimulated AMPK via changing the ratio of intracellular AMP/ATP. (A) Chromatogram of AMP, ADP and ATP by using FPLC. (B) Intracellular AMP, ADP and ATP levels of control, ATCAA-10 (10 $\mu \mathrm{M})$, and PD98059 (100 $\mu \mathrm{M})$ in HeLa cells (n=3); bars, SD. PD98059 was used as a positive control. (C) The ratio of intracellular AMP/ATP of ATCAA-10 based on (B). (D) AMPK enzyme activity assay in cell-free system. ATCAA-10 $(10 \mu \mathrm{M})$ was used to test the induction of AMPK. AMP $(10 \mu \mathrm{M})$ was used as a positive control. 
A

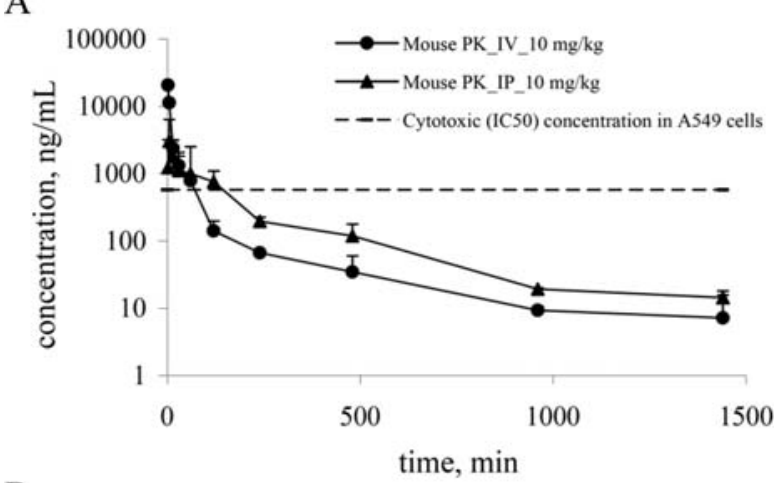

B

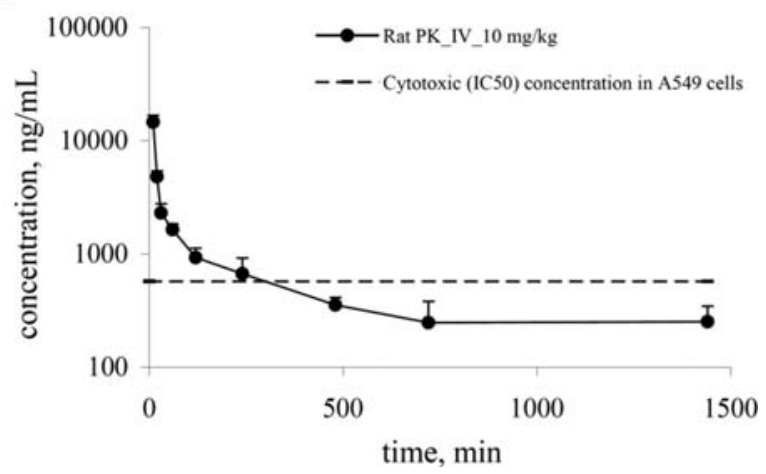

Figure 3. Pharmacokinetic studies of ATCAA-10. (A) Concentration-time curve of ATCAA-10 in ICR mice ( $\mathrm{n}=3)$; bars, SD. ATCAA-10 was administered $10 \mathrm{mg} / \mathrm{kg}$ by i.v.and i.p. Concentration-time curve of ATCAA-10 in SD rats $(\mathrm{n}=4)$; bars, $\mathrm{SD}$ (B) The SD rat dose was $10 \mathrm{mg} / \mathrm{kg}$ i.v. Dashed line indicates that cytotoxic $\mathrm{IC}_{50}$ value obtained in $\mathrm{A} 549$ cancer cell line in vitro. The $\mathrm{IC}_{50}$ value was $1.1 \mu \mathrm{M}(574 \mathrm{ng} / \mathrm{ml})$.

Table III. The pharmacokinetic parameters of ATCAA-10 $(10 \mathrm{mg} / \mathrm{kg})$ in mice and rats.

\begin{tabular}{|c|c|c|c|c|}
\hline \multirow[b]{2}{*}{$\begin{array}{l}\text { Parameter } \\
\text { Unit }\end{array}$} & \multicolumn{4}{|c|}{$\begin{array}{l}\text { In vivo, pharmacokinetic parameters of } \\
\text { ATCAA-10 }\end{array}$} \\
\hline & $\begin{array}{c}\text { AUC } \\
\mathrm{h}^{*} \mathrm{mg} / \mathrm{ml}\end{array}$ & $\begin{array}{l}\mathrm{t}_{1 / 2} \\
\min \end{array}$ & $\begin{array}{c}V_{\mathrm{ss}} \\
1 / \mathrm{kg}\end{array}$ & $\begin{array}{c}\mathrm{CL} \\
\mathrm{ml} / \mathrm{min} / \mathrm{kg}\end{array}$ \\
\hline Mice & 4.9 & 311 & 2.9 & 34 \\
\hline Rats & 17.3 & 477 & 4.1 & 8.3 \\
\hline
\end{tabular}

administration for mice. Most importantly, the PK studies by i.p. injection also demonstrated that plasma concentrations above the in vitro effective concentration obtained in the cytotoxicity assays in A549 cancer cells could be achieved.

Similar to mice PK, ATCAA-10 also exhibited favorable PK properties in rats, exhibiting a long half-life (477 $\mathrm{min}$ ), moderate volume of distribution at steady state $(4.1 \mathrm{l} / \mathrm{kg})$, and low clearance $(8.3 \mathrm{ml} / \mathrm{min} / \mathrm{kg})$, with low hepatic extraction ratio (ER=0.28, if the hepatic plasma flow rate is $30 \mathrm{ml} / \mathrm{min} / \mathrm{kg}$ in rats).

ATCAA-10 inhibited lung cancer A549 xenograft growth. Lung cancer A549 xenografts were allowed to reach a volume

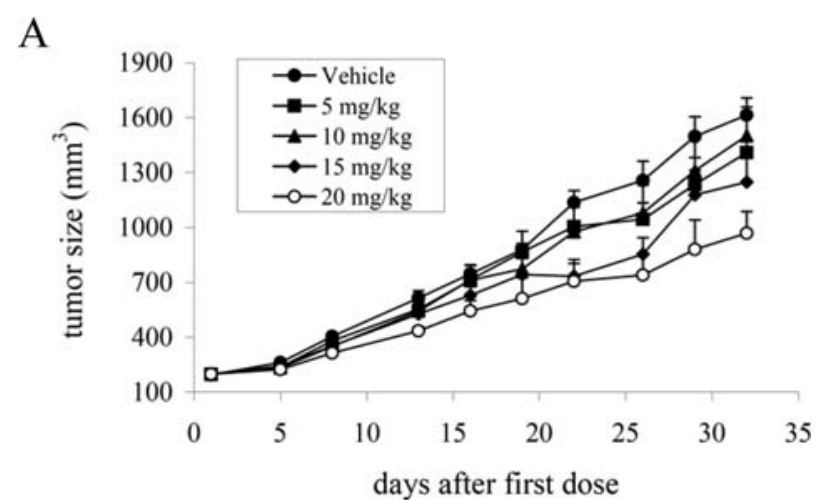

B

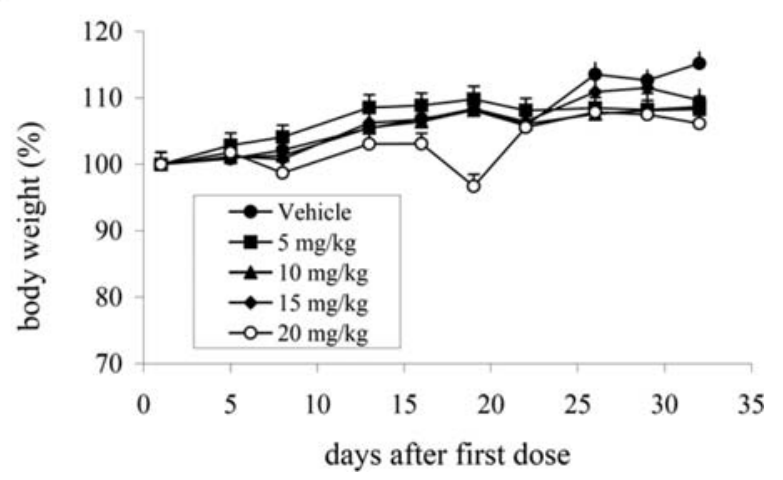

Figure 4. In vivo efficacy of ATCAA-10. Tumor cells were s.c. implanted in nude mice. ATCAA-10 was administered after tumor formation (150$200 \mathrm{~mm}^{3}, \mathrm{n}=6-8$ ) (A) Human lung A549 xenografts were treated with 5, 10, 15 and $20 \mathrm{mg} / \mathrm{kg}$ i.p.; bars, SE. (B) Body weights were measured twice a week. The percentage of body weights were determined by comparing with the body weight on day 1 ; bars, SD.

of $200 \mathrm{~mm}^{3}$ and the tumor-bearing mice were treated with ATCAA-10 by i.p. administration at the indicated doses. As shown in Fig. 4A, tumor volumes in the control group increased to $1615 \pm 244 \mathrm{~mm}^{3}$ over the 31 day study duration. ATCAA-10 elicited $46 \%$ tumor growth inhibition (TGI) at $20 \mathrm{mg} / \mathrm{kg}$ treatment, decreasing tumor volumes to $968 \pm 358 \mathrm{~mm}^{3}$. Body weight measurements (Fig. 4B), to monitor toxicity, indicated that ATCAA-10 did not significantly reduce body weights at the effective dose.

ATCAA-10 did not cause hyperglycemia at a single dose in rats or repeated-doses in mice. Inhibitors affecting the PI3K/Akt/ mTOR pathway are often associated with hyperglycemia and hyperinsulinemia due to their integral role in insulin signaling (21). To test if hyperglycemia or hyperinsulinemia was caused by ATCAA-10, whole blood glucose and plasma insulin were measured when the rat PK studies were performed. Fig. 5A shows that a single dose $(10 \mathrm{mg} / \mathrm{kg})$ of ATCAA-10 in rats did not cause a significant change in whole blood glucose levels. The same trend was also observed in plasma insulin levels (Fig. 5B), suggesting that ATCAA-10 did not induce hyperglycemia and hyperinsulinemia by single dose in a short-term study $(24 \mathrm{~h})$. Though single dose failed to induce hyperglycemia, cancer patients are treated for a prolonged duration. Blood glucose levels in nude mice treated long-term with ATCAA-10 (twice a week, 31 days) were $194 \pm 47,198 \pm 53,199 \pm 44,211 \pm 44$, and $223 \pm 61 \mathrm{mg} / \mathrm{dl}$ in 
A

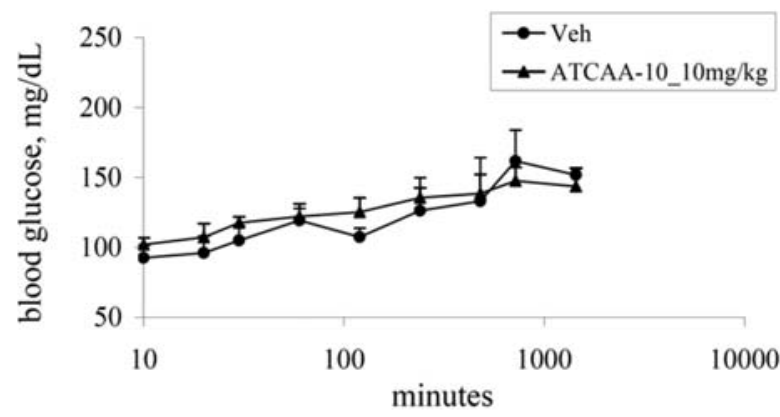

B

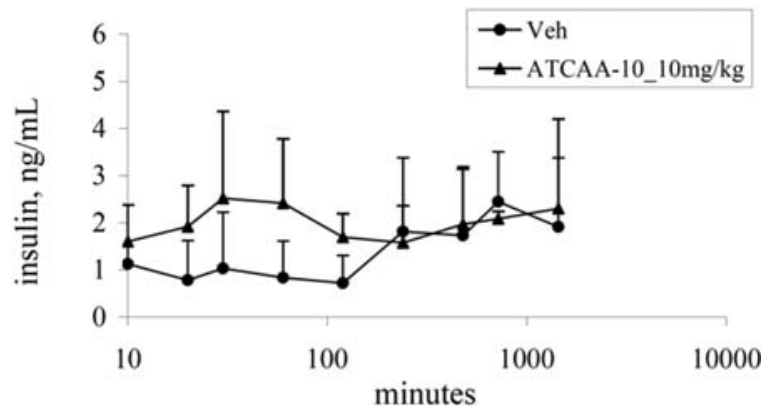

Figure 5. Hyperglycemia and hyperinsulinemia test. (A) Blood glucose levels of ATCAA-10 with $10 \mathrm{mg} / \mathrm{kg}$ i.v. in SD rats (B) Plasma insulin levels of ATCAA-10 treatment. Plasma insulin was determined using ELISA kit Vehicle was DMSO/PEG300 (1/4). N=4; bars, SD.

control, 5, 10, 15, and $20 \mathrm{mg} / \mathrm{kg}$, respectively. These data indicate that ATCAA-10 did not induce hyperglycemia after repeated doses over 31 days in mice.

\section{Discussion}

ATCAA-10 exhibited potent antitumor activities against a spectrum of cancer cell lines with $\mathrm{IC}_{50}$ values in the range of 0.4-1.8 $\mu \mathrm{M}$ (Table I). Since resistance to prolonged treatment due to over-expression of P-gp is a major problem, studies were also conducted to determine whether ATCAA-10 is a substrate of P-gp. Our data and other publications demonstrated that the overexpression of P-gp in MES-SA/Dx5 results in a high resistance factor and varied dose-response curve for vinblastine (22). However, ATCAA-10 showed similar activity in both MES-SA and MES-SA/Dx 5 cell lines and did not cause a varied dose-response curve, suggesting that ATCAA-10 may circumvent P-gp-mediated drug resistance. These data suggest that resistance may not be a major problem for ATCAA-10.

We found that dephosphorylation of Akt by ATCAA-10 occurred not only in A549 and HeLa cells (in this study), but also in PTEN-mutated prostate cancer cell lines, such as LNCaP and PC-3 (data not shown). These data confirmed that ATCAA-10 consistently, regardless of the cancer type, targets PI3K/Akt pathway. The mechanism for the dephosphorylation of Akt by ATCAA-10 was investigated in this study. In our earlier studies (20), we speculated that ATCAA-10 target lysophosphatidic acid (LPA) receptors to inhibit Akt, as the ATCAA were originally designed as mimics of LPA. However, ATCAA-10 did not target the LPA receptors leading us to examine other upstream RTKs and GPCRs. We excluded IGF-1R, EGFR, FGFR, and PDGFR as targets of ATCAA-10. Future studies will include more receptor tyrosine kinases and GPCRs. PDK1 is the direct upstream activation of Akt, and ATCAA-10 did not inhibit PDK1. The PI3K and Akt themselves were also tested, but ATCAA-10 failed to effectively inhibit their activities, suggesting that the target of ATCAA-10 may be obscure.

Hyperglycemia has been reported as an on-target sideeffect for agents targeting the PI3K/Akt pathway in animal models (21) and has also been observed in patients treated with triciribine phosphate (23). Although ATCAA-10 is involved in PI3K/Akt pathway, a hyperglycemic effect was not observed after a signal dose $(10 \mathrm{mg} / \mathrm{kg})$ of ATCAA-10 in rats or repeated dosing in mice (twice a week for 31 days). These data suggest that ATCAA-10 may circumvent this sideeffect.

ATCAA-10 stimulated phospho-AMPK in both A549 and HeLa cell lines. We confirmed that ATCAA-10 induced phospho-AMPK by depleting ATP and changing the intracellular AMP/ATP ratio, but by directly targeting AMPK. The upstream of AMPK, LKB1 kinase, can phosphate and activate AMPK. However, both A549 and HeLa cells are LKB1-null (24), indicating that ATCAA-10 stimulated phospho-AMPK independent of its upstream kinase LKB1. Since AMPK is considered as a negative regulator of proliferation that attenuates cancer, we hypothesize that part of ATCAA-10's anti-proliferative effects emanate from AMPK activation. AICAR and phenformin are often experimentally examined for stimulation of AMPK and subsequent inactivation of Akt (25). However, the concentration of AICAR or phenformin needed to induce these effects is in millimolar range (i.e., 1000-fold higher than ATCAA-10). It remains controversial whether Akt regulates AMPK activity or vice versa. Recent evidence indicates that activated AMPK induces the expression of p21, p27, and p53 proteins and suppresses the PI3K/Akt pathway (26). Conversely, Akt is claimed to regulate the intracellular ATP levels and act as a negative regulator of AMPK (27). Both activation of AMPK and deactivation of Akt may contribute to tumor growth inhibition observed after treatment with ATCAA-10. In further studies, the use of Akt or AMPK antisense experiments combining ATCAA-10 treatment may help to elucidate the relationship between Akt and AMPK.

ATCAA-10 exhibited acceptable PK properties in mice and rats. By the i.p. route in mice, plasma concentrations exceeded $574 \mathrm{ng} / \mathrm{ml}\left(\mathrm{IC}_{50}\right.$ value in A549 cell line in vitro) for more than two hours at a dose of $10 \mathrm{mg} / \mathrm{kg}$, providing proof that sufficient systemic exposure could be and was achieved during tumor xenograft studies in mice. Since ATCAA-10 exhibited favorable PK properties in rats, we also tested the oral bioavailability of ATCAA-10. Unfortunately, the oral bioavailability was only $4.4 \%$ after a dose at $10 \mathrm{mg} / \mathrm{kg}$ in rats (data not shown), suggesting that ATCAA-10 may have low permeability, be susceptible to intestinal metabolism or have limited solubility. Permeability studies using Caco- 2 cells confirmed that ATCAA-10 has an extremely low permeability (Papp is $<1 \times 10^{-6} \mathrm{~cm} / \mathrm{s}, \mathrm{A} \rightarrow \mathrm{B}$, data not shown), suggesting further modifications on the chemical scaffold of ATCAA-10 will be necessary to achieve acceptable oral bioavailability. 
These studies showed that ATCAA-10 inhibited the growth of A549 lung tumor xenograft via inhibition of PI3K/Akt pathway and activation of AMPK pathway. We also found that ATCAA-10 exhibited acceptable PK properties for tumor growth inhibition. In efficacy, ATCAA-10 treatments were able to reduce the growth of tumors in animals in a dosedependent manner, and achieved $46 \%$ of TGI at $20 \mathrm{mg} / \mathrm{kg}$ group. These studies provide initial in vitro and in vivo proof of concept that ATCAAs or optimized derivatives thereof modulate the activity of the PI3K/Akt and AMPK pathways and hold promise as a novel approach to the treatment of drug-resistant cancer.

\section{Acknowledgments}

We thank Terrence A. Costello, Katie N. Kail and Stacey L. Barnett for providing technical support for animal studies at GTx Inc.

\section{References}

1. Engelman JA, Luo J and Cantley LC: The evolution of phosphatidylinositol 3-kinases as regulators of growth and metabolism. Nat Rev Genet 7: 606-619, 2006.

2. Wymann MP, Zvelebil M and Laffargue M: Phosphoinositide 3-kinase signalling - which way to target? Trends Pharmacol Sci 24: 366-376, 2003

3. Feng J, Park J, Cron P, Hess D and Hemmings BA: Identification of a PKB/Akt hydrophobic motif Ser-473 kinase as DNAdependent protein kinase. J Biol Chem 279: 41189-41196, 2004.

4. Vivanco I and Sawyers CL: The phosphatidylinositol 3-Kinase AKT pathway in human cancer. Nat Rev Cancer 2: 489-501, 2002

5. Grunwald V, De Graffenried L, Russel D, Friedrichs WE, Ray RB and Hidalgo M: Inhibitors of mTOR reverse doxorubicin resistance conferred by PTEN status in prostate cancer cells. Cancer Res 62: 6141-6145, 2002.

6. Cohen P and Frame S: The renaissance of GSK3. Nat Rev Mol Cell Biol 2: 769-776, 2001

7. Frame S and Cohen P: GSK3 takes centre stage more than 20 years after its discovery. Biochem J 359: 1-16, 2001.

8. Dan HC, Sun M, Yang L, et al: Phosphatidylinositol 3-kinase/ Akt pathway regulates tuberous sclerosis tumor suppressor complex by phosphorylation of tuberin. J Biol Chem 277: 35364-35370, 2002.

9. Mita MM, Mita A and Rowinsky EK: The molecular target of rapamycin (mTOR) as a therapeutic target against cancer. Cancer Biol Ther 2: S169-S177, 2003

10. Gunn RM and Hailes HC: Insights into the PI3-K-PKB-mTOR signalling pathway from small molecules. J Chem Biol 1: 49-62, 2008
11. Hardie DG and Carling D: The AMP-activated protein kinasefuel gauge of the mammalian cell? Eur J Biochem 246: 259-273, 1997.

12. Woods A, Johnstone SR, Dickerson K, et al: LKB1 is the upstream kinase in the AMP-activated protein kinase cascade. Curr Biol 13: 2004-2008, 2003

13. Hardie DG, Salt IP, Hawley SA and Davies SP: AMP-activated protein kinase: an ultrasensitive system for monitoring cellular energy charge. Biochem J 338: 717-722, 1999.

14. Kemp BE, Stapleton D, Campbell DJ, et al: AMP-activated protein kinase, super metabolic regulator. Biochem Soc Trans 31: 162-168, 2003.

15. Inoki K, Zhu T and Guan KL: TSC2 mediates cellular energy response to control cell growth and survival. Cell 115: 577-590, 2003.

16. Kimura N, Tokunaga C, Dalal S, et al: A possible linkage between AMP-activated protein kinase (AMPK) and mammalian target of rapamycin (mTOR) signalling pathway. Genes Cells 8: 65-79, 2003.

17. Luo Z, Saha AK, Xiang X and Ruderman NB: AMPK, the metabolic syndrome and cancer. Trends Pharmacol Sci 26: 69-76, 2005.

18. Hadad SM, Fleming S and Thompson AM: Targeting AMPK: a new therapeutic opportunity in breast cancer. Crit Rev Oncol Hematol 67: 1-7, 2008.

19. Motoshima H, Goldstein BJ, Igata M and Araki E: AMPK and cell proliferation - AMPK as a therapeutic target for atherosclerosis and cancer. J Physiol 574: 63-71, 2006.

20. Lu Y, Wang Z, Li CM, et al: Synthesis, in vitro structureactivity relationship, and in vivo studies of 2-arylthiazolidine-4carboxylic acid amides as anticancer agents. Bioorg Med Chem 18: 477-495, 2010.

21. Crouthamel MC, Kahana JA, Korenchuk S, et al: Mechanism and management of AKT inhibitor-induced hyperglycemia. Clin Cancer Res 15: 217-225, 2009.

22. Traunecker HC, Stevens MC, Kerr DJ and Ferry DR: The acridonecarboxamide GF120918 potently reverses P-glycoprotein-mediated resistance in human sarcoma MES-Dx5 cells. Br J Cancer 81: 942-951, 1999.

23. Hoffman K, Holmes FA, Fraschini G, et al: Phase I-II study: triciribine (tricyclic nucleoside phosphate) for metastatic breast cancer. Cancer Chemother Pharmacol 37: 254-258, 1996.

24. Hurley RL, Anderson KA, Franzone JM, Kemp BE, Means AR and Witters LA: The $\mathrm{Ca}^{2+} /$ calmodulin-dependent protein kinase kinases are AMP-activated protein kinase kinases. J Biol Chem 280: 29060-29066, 2005

25. King TD, Song L and Jope RS: AMP-activated protein kinase (AMPK) activating agents cause dephosphorylation of Akt and glycogen synthase kinase-3. Biochem Pharmacol 71: 1637-1647, 2006.

26. Rattan R, Giri S, Singh AK and Singh I: 5-Aminoimidazole-4carboxamide-1-beta-D-ribofuranoside inhibits cancer cell proliferation in vitro and in vivo via AMP-activated protein kinase. J Biol Chem 280: 39582-39593, 2005.

27. Hahn-Windgassen A, Nogueira V, Chen CC, Skeen JE, Sonenberg N and Hay N: Akt activates the mammalian target of rapamycin by regulating cellular ATP level and AMPK activity. J Biol Chem 280: 32081-32089, 2005. 